\title{
The clinical significance of serum oxidative stress biomarkers in breast cancer females
}

\author{
Corresponding author: \\ Prof.Dr. Taha I. Hewala; Department \\ of Radiation Sciences, Medical Re- \\ search Institute, Alexandria \\ University, 165 Horria Avenue, \\ El Hadara, Alexandria, 21561, Egypt, \\ tel.: +203-4229118 \\ fax: $+203-4283719$, \\ e-mail: tahahewala@alex-mri.edu.eg
}

Medical Research Journal 2019; Volume 4, Number 1, 1-7 DOI: 10.5603/MRJ.a2018.0039 Copyright (c) 2019 Via Medica ISSN 2451-2591

\begin{abstract}
Background: This study was conducted to evaluate the role of some serum oxidative stress biomarkers for breast cancer diagnosis, incidence and monitoring the effects of surgery and chemotherapy. Methods: A total of 35 breast cancer patients (before surgery, after two weeks of surgery and after 6 cycles of chemotherapy) and 35 normal healthy controls were analyzed for serum oxidative stress markers including total antioxidant capacity (TAC), malondialdehyde (MDA), total, reduced (GSH) and oxidized (GSSG) glutathione and glutathione redox status (GSH/GSSG).

Results: The serum levels of MDA and GSSG were significantly higher in breast cancer patients than controls. The serum levels of GSH, TAC and GSH/GSSG ratio were significantly lower in breast cancer patients than controls. After surgery, the serum levels of MDA and GSSG were significantly decreased, while the serum levels of GSH were significantly increased, compared with their levels before surgery. Six cycles of chemotherapy showed the non-significant effect on the serum levels of the assayed biomarkers. ROC curve analysis demonstrated that MDA and GSH were superior to the GSH/GSSG ratio, TAC and GSSG. Increased levels of MDA and GSSG and reduced levels of GSH, TAC and GSH/GSSG ratio were found to significantly increase the risk of breast cancer.

Conclusions: All of the assayed biomarkers can be used for prediction of breast cancer with MDA and GSH being superior to the others. MDA, GSH and GSSG were able to monitor the effect of surgery. All of the assayed biomarkers were found to be associated with breast cancer risk. None of the assayed biomarkers was able to predict the effect of chemotherapy.

Key words: Breast cancer, MDA, TAC, GSH, GSSG, Glutathione redox status
\end{abstract}

\section{Introduction}

Reactive oxygen species (ROS) such as superoxide radical, hydroxyl radical, and hydrogen peroxide are metabolic by-products leaking from the complexes I and III of the mitochondrial respiratory chain [1]. Oxidative stress is considered to be involved in the pathophysiology of all cancers including breast cancer [2, 3].

Breast cancer cells are subjected to a high level of oxidative stress, both intracellular and extracellular. To ensure survival, cancer cells must acquire special adaptive mechanisms that counteract the toxic effects of free radicals exposure. These mechanisms may involve the activation of redox-sensitive transcription factors and the increased expression of antioxidant enzymes and antiapoptotic proteins. Moreover, it was revealed that different breast cancer cell types show different intracellular antioxidant capacities that may determine their ability to resist radiotherapy and chemotherapy [4].

The involvement of oxidative stress in breast carcinogenesis has not been extensively documented [5]. Panis et al. [6] reported that although a growing number of studies have focused on the relationship between breast cancer and oxidative stress, none of the previous reports determined the impact of the systemic oxidative stress status on patients with primary breast tumours, as well as if its removal could change the oxidative profiling of plasma.

One of the possible clinical applications of oxidative stress status in cancer is the use of oxidative stress markers as tumour markers [7]. The current study examined the extent of lipid peroxidation, total antioxidant capacity and the status of glutathione in serum of breast cancer patients to investigate the role of these biomarkers in the etiology, prediction, risk and monitoring the 
effects of surgery and chemotherapy on breast cancer patients in an attempt to improve the clinical outcome of those patients.

\section{Subjects and methods}

Seventy females were enrolled in this case-control study. Females were divided into two groups. Group I (breast cancer patients group) which included 35 female patients with breast invasive ductal carcinoma of clinical stages II and III [8] (recently detected, not underwent surgery or receiving chemotherapy). Their mean age was $43.73 \pm 12.2$ years. Patients were recruited from the Department of Experimental and Clinical Surgery of the Medical Research Institute, Alexandria University, Egypt in the period from January 2018 till June 2018. Group II (normal healthy control group): It included 35 apparently normal healthy female volunteers of comparable age (42.18 \pm 11.05$)$, menstrual cycle and socioeconomic status as patients.

After having approval from the ethics committee, Medical Research Institute, Alexandria University, Egypt, signed informed consents were obtained from all subjects who agreed to participate in this study. Each patient underwent full history recording, thorough clinical examination, routine laboratory investigations including complete blood count (CBC), mammography of breast and ultrasonography of abdomen and liver, radiological investigations including $X$-ray chest, CT scan and bone scan when needed and fine needle aspiration cytology (FNAC) of breast mass to establish the pathological diagnosis in the patients.

The clinicopathologic data were obtained from patients' pathology reports. The collected data included tumour size, tumour pathological grade, axillary lymph node involvement, vascular invasion and status of estrogen receptor (ER) and progesterone receptor (PR). Each breast cancer patient's clinical stage was determined by the oncologist according to the tumour-nodes-metastasis (TNM) classification system [8].

All 35 breast cancer patients were subjected to modified radical mastectomy (MRM) surgery [9]. The patients received adjuvant combination chemotherapy [5-Fluorouracil, Adriamycin and Cyclophosphamide (FAC)] [10] for 6 cycles. After 6 cycles of chemotherapy, breast cancer patients were re-evaluated clinically, laboratory and radiologically to evaluate the clinical response.

\section{Laboratory Investigations}

Five-milliliter blood sample was collected once from normal healthy female volunteers and from breast cancer patients before surgery, after 2 weeks of surgery and after 6 cycles of chemotherapy. Immediately after withdrawing, blood samples were allowed to coagulate and centrifuged for 20 minutes at $3500 \mathrm{rpm}$. The separated serum samples were aliquoted, frozen at $-80{ }^{\circ} \mathrm{C}$, and stored until assayed. After thawing, each serum aliquot was assayed only once. Determination of serum levels of MDA, total antioxidant capacity, total and reduced forms of glutathione were carried out at Radiation Sciences Department, Medical Research Institute, Alexandria University, Egypt. While the serum levels of oxidized glutathione and glutathione redox status were estimated mathematically.

\section{Determination of serum malondialdehyde concentration}

Malondialdehyde (MDA) is the end product of lipid peroxidation. MDA was assayed using a ready-for-use colourimetric kit (Biodiagnostic, Egypt). MDA was quantified by its reaction with the exogenously added thiobarbituric acid (TBA) that reacts with MDA in acidic medium at a temperature of $95^{\circ} \mathrm{C}$ for $30 \mathrm{~min}$ to form thiobarbituric acid (TBA) reactive product. The absorbance of the resultant pink product was measured at $534 \mathrm{~nm}$. The absorbance of each serum sample was read against a blank (which contained only TBA) and a standard (which contained MDA of conc. $10 \mathrm{nmo-}$ $\mathrm{le} / \mathrm{ml})$. In each serum sample, the concentration of MDA was calculated according to the equation:

\section{MDA $(\mathrm{nmole} / \mathrm{ml})=($ Abs of serum $/$}

(Abs of standard) $* 10$

\section{Determination of serum total antioxidant capacity}

The degree of oxidative stress was assessed by determining total antioxidant activity using a ready-for-use colourimetric kit (Biodiagnostic, Egypt). The principle of the method depends on the reaction of antioxidants in the sample with a defined amount of exogenously added $\mathrm{H}_{2} \mathrm{O}_{2}$. The antioxidants in the sample eliminate a certain amount of the added hydrogen peroxide. The residual $\mathrm{H}_{2} \mathrm{O}_{2}$ is determined colourimetrically by an enzymatic reaction involving the conversion of the exogenously added 3 , 5, dichloro-2-hydroxyl benzenesulfonate to a pink coloured product. The absorbance of each sample was read at $505 \mathrm{~nm}$ against a blank in which the sample was replaced with distilled water. In each sample, the total antioxidant concentration was calculated according to the equation:

Total antioxidant concentration ( $\mathrm{mM} / \mathrm{L}$ )

$=($ Abs of blank-Abs of sample $) * 3.33$

\section{Determination of serum levels of total glutathione}

The enzymatic method described by Griffith [11] was used to measure the levels of total glutathione. This is a sensitive and specific enzymatic method which depends on the oxidation of reduced glutathione (GSH) 
by 5,5-dithiobis-2-nitrobenzoic acid (DTNB) to yield oxidized glutathione (GSSG) and 5-thio-2-nitrobenzoic acid (TNB). Oxidized GSSG is reduced enzymatically by the action of glutathione reductase and NADPH to regenerate $\mathrm{GSH}$ which reacts again. The rate of TNB formation is monitored at $340 \mathrm{~nm}$ and is proportional to the sum of GSH and GSSG present in the sample.

Total glutathione $(\mathrm{U} / \mathrm{L})=4019 \times \Delta 340 \mathrm{~nm} / \mathrm{min}$

\section{Determination of serum levels of reduced glutathione (GSH)}

The level of reduced glutathione in serum was assessed using a ready-for-use colourimetric kit (Biodiagnostic, Egypt). The method is based on the reduction of 5,5 dithiobis -2-nitrobenzoic acid (DTNB) by glutathione (GSH) to produce a yellow compound. The reduced chromogen directly proportional to GSH concentration and its absorbance can be measured at $405 \mathrm{~nm}$.

\section{Serum level of $\mathrm{GSH}(\mathrm{mg} / \mathrm{dl})$ \\ $=($ A"sample" $) \star 66.66$}

\section{Estimation of serum levels of oxidized glutathione (GSSG):}

Oxidized glutathione (GSSG) was obtained by subtracting the values of reduced glutathione (GSH) from the values of total glutathione (tGSH) [12].

$$
\text { GSSG = tGSH }- \text { GSH }
$$

\section{Estimation of serum glutathione redox status (GSH/GSSG)}

The most widely used indicator of the glutathione redox status (GSH/GSSG) of the cells is according to Kirlin et al. [13]:

$$
\begin{gathered}
\text { Redox Status }=(\text { Serum level of GSH) } / \\
/(\text { Serum level of GSSG) }
\end{gathered}
$$

Where [GSH] and [GSSG] are molar concentration of reduced and oxidized glutathione; respectively.

\section{Statistical Analysis}

The statistical analyses were carried out using the Predictive Analytics software (PASW statistics 18). The Non-parametric Mann-Whitney U-test was used for studying differences between breast cancer patients group and control group regarding serum levels of total antioxidant capacity (TAC), malondialdehyde (MDA), total, reduced (GSH) and oxidized (GSSG) glutathione and glutathione redox status (GSH/GSSG). The diagnostic values of assayed parameters were compared using the Receiver Operating Characteristic (ROC) curve analysis. The cut-off point for each biomarker was determined according to the best discrimination between patients and controls regarding optimal values of sensitivity and specificity using the ROC curve. Spearman correlation was carried out to explore the possible correlation between different biomarkers and clinicopathological data. Odd's ratio with a 95\% confidence interval $(95 \% \mathrm{Cl})$ was calculated using the same program. P-values $<0.05$ were accepted as significant.

\section{Results}

Serum levels of oxidative stress biomarkers in the controls and breast cancer patients before surgery, after two weeks of surgery and after 6 cycles of chemotherapy.

Table 1 showed oxidative stress biomarkers in serum of normal healthy control females and breast cancer females before surgery, after two weeks of surgery and after six cycles of chemotherapy. Serum levels of MDA and GSSG were significantly elevated in breast cancer patients group before surgery compared with

\begin{tabular}{|c|c|c|c|c|}
\hline \multirow[t]{2}{*}{ Serum biomarkers } & \multirow[t]{2}{*}{$\begin{array}{l}\text { Control group } \\
\quad(n=35)\end{array}$} & \multicolumn{3}{|c|}{$\begin{array}{l}\text { Breast cancer patients group } \\
\qquad(\mathrm{n}=35)\end{array}$} \\
\hline & & BS & $2 \mathrm{~W}$ & $6 \mathrm{C}$ \\
\hline $\begin{array}{l}\text { MDA } \\
\text { (nmole/ml) }\end{array}$ & $11.10 \pm 0.44$ & $20.01 \pm 0.37^{a}$ & $12.27 \pm 0.29^{b}$ & $13.56 \pm 0.33$ \\
\hline $\mathrm{TAC}(\mathrm{mM} / \mathrm{l})$ & $1.26 \pm 0.49$ & $0.78 \pm 0.46^{a}$ & $0.90 \pm 0.40^{a}$ & $0.83 \pm 0.38^{a}$ \\
\hline GSSG(mg/dl) & $9.23 \pm 0.21$ & $10.39 \pm 2.22^{a}$ & $9.64 \pm 2.91^{b}$ & $10.29 \pm 2.98$ \\
\hline GSH (mg/dl) & $21.71 \pm 1.07$ & $15.85 \pm 1.08^{a}$ & $17.14 \pm 2.13^{a, b}$ & $13.61 \pm 4.01^{a}$ \\
\hline $\begin{array}{l}\text { Glutathione Redox status } \\
\text { (GSH/GSSG) }\end{array}$ & $2.38 \pm 0.47$ & $1.84 \pm 0.14^{a}$ & $1.57 \pm 0.16^{a}$ & $1.54 \pm 0.13^{a}$ \\
\hline
\end{tabular}

Table 1. Serum levels (Mean \pm SE) of oxidative stress biomarkers in the controls and breast cancer patients before surgery, after 2 weeks of surgery and after 6 cycles of chemotherapy

$\mathrm{N}$ - sample size; $\mathrm{BS}$ - before surgery; $2 \mathrm{~W}$ - after two weeks of surgery; $6 \mathrm{C}$ - after six cycles of chemotherapy; a - significance compared with control group; b - significance compared with breast cancer group before surgery; c - significance compared with breast cancer group after two weeks of surgery; significance was considered at $p$-value $<0.05$. 
the control group. The serum levels of MDA and GSSG were significantly decreased in breast cancer patients group after two weeks of surgery compared with their levels before surgery. A non-significant difference was found in serum levels of MDA and GSSG after 6 cycles of chemotherapy compared with their levels after two weeks of surgery.

Table 1 also showed that serum levels of TAC, GSH and glutathione redox status (GSH/GSSG) were significantly decreased in breast cancer females before surgery compared with the control group. The serum levels of reduced glutathione in breast cancer patients group after two weeks of surgery were significantly elevated compared with their levels before surgery. After six cycles of chemotherapy, none of these biomarkers significantly differed from its level after two weeks of surgery.

\section{Comparison between serum levels of oxidative stress biomarkers as diagnostic markers in breast cancer patients using the Receiver Operating Characteristic (ROC) curve analysis}

As shown in Table 2 and Figure 1, the ROC curve analysis was used in the present study to compare the diagnostic values of serum oxidative stress biomarkers depending on the area under the ROC curve (AUC). The higher AUC corresponds to a better diagnostic test. Serum levels of MDA showed a significant AUC $(96 \%),(p=0.001)$, with sensitivity $(90 \%)$ and specificity $(80 \%)$ at a cut-off value $(13.81 \mathrm{nmol} / \mathrm{ml})$. Serum levels of TAC showed a significant AUC (76.9\%), $(p=0.005)$, with sensitivity $(85.7 \%)$ and specificity $(57.7 \%)$ at a cut-off value $(0.90 \mathrm{mM} / \mathrm{L})$. Serum levels of oxidized glutathi-
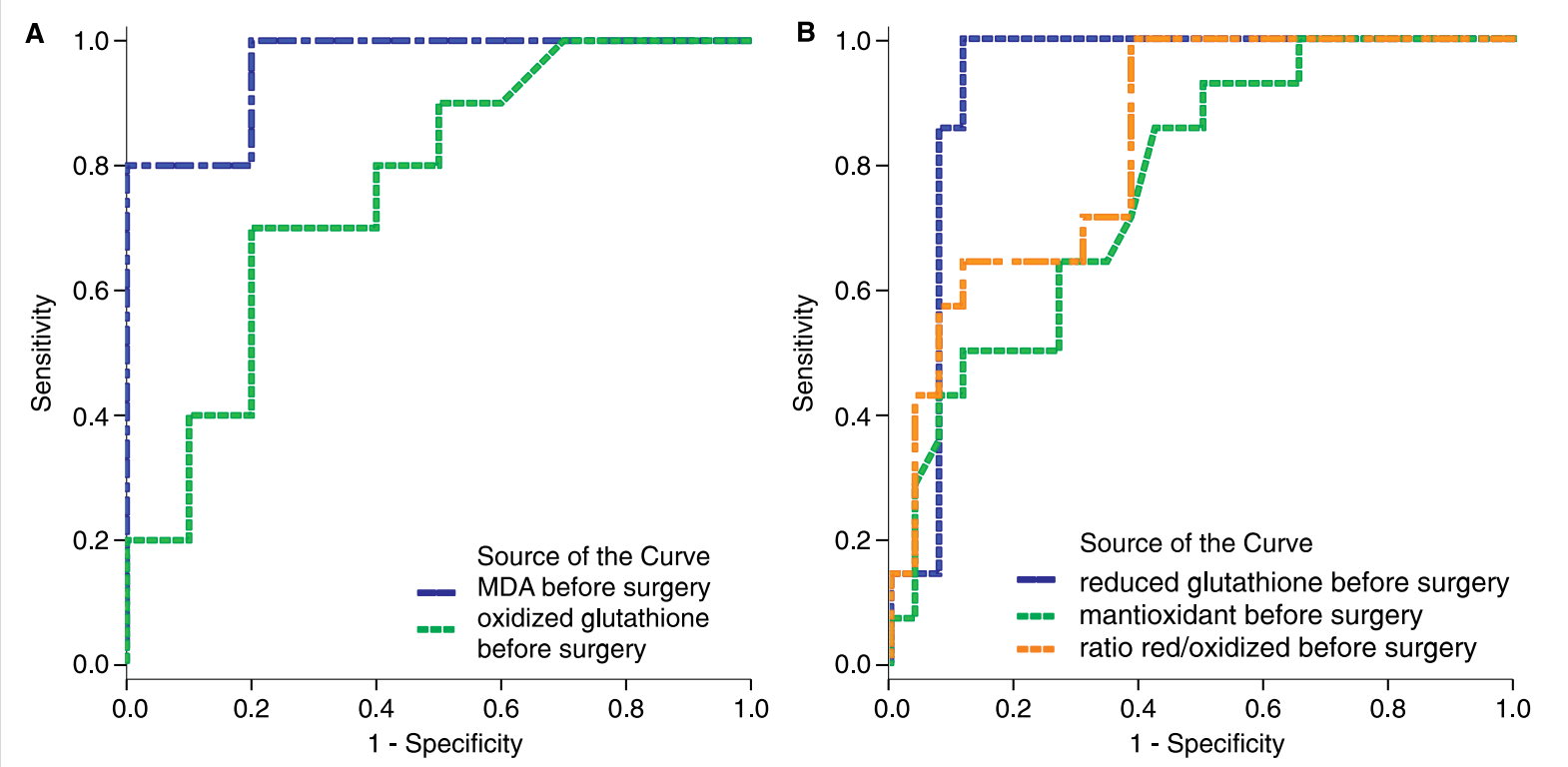

Figure 1. The ROC curve analysis of the assayed biomarkers for prediction of breast cancer

Table 2. The ROC curve-based characteristics for assayed serum markers in breast cancer patients before surgery

\begin{tabular}{lccccc}
\hline Variables & $\begin{array}{c}\text { The area under } \\
\text { the curve (\%) }\end{array}$ & P-value & Cut-off value & $\begin{array}{c}\text { Sensitivity } \\
\%\end{array}$ & $\begin{array}{c}\text { Specificity } \\
\%\end{array}$ \\
\hline $\begin{array}{l}\text { MDA } \\
(\mathrm{nmol} / \mathrm{ml})\end{array}$ & 96.0 & $0.001^{*}$ & 13.81 & 90 & 80 \\
$\begin{array}{l}\mathrm{TAC} \\
(\mathrm{mM} / \mathrm{l})\end{array}$ & 76.9 & $0.005^{*}$ & 0.90 & 85.7 & 57.7 \\
$\begin{array}{l}\mathrm{GSSG} \\
(\mathrm{mg} / \mathrm{ml})\end{array}$ & 70.5 & $0.035^{*}$ & 9.9 & 84.6 & 80.6 \\
$\begin{array}{l}\mathrm{GSH} \\
(\mathrm{mg} / \mathrm{ml})\end{array}$ & 92.9 & $0.000^{*}$ & 17.30 & 92.9 & 100 \\
$\begin{array}{l}\text { Glutathione Redox } \\
\text { status GSH/GSSG) }\end{array}$ & 82.1 & $0.001^{*}$ & 1.70 & 9 & 76.9
\end{tabular}

*Significance was considered at $p$-value $<0.05$. 
Table 3. The association of serum assayed biomarkers with the risk of breast cancer incidence

\begin{tabular}{|c|c|c|c|c|c|c|}
\hline Assayed Biomarker & & $\begin{array}{c}\text { breast cancer } \\
\text { group } \\
(\mathbf{n}=35)\end{array}$ & $\begin{array}{l}\text { control group } \\
(n=35)\end{array}$ & $\begin{array}{l}\text { Odds ratio } \\
\text { (OR) }\end{array}$ & $95 \% \mathrm{Cl}$ & $p$-value \\
\hline \multirow{2}{*}{$\begin{array}{l}\text { MDA } \\
\text { (nmole/ml) }\end{array}$} & $<13.81 \AA$ & 13 & 30 & 10.15 & $1.69-11.06$ & $P=0.002$ * \\
\hline & $\geq 13.81$ & 22 & 5 & & & \\
\hline \multirow{2}{*}{$\begin{array}{l}\text { TAC } \\
(\mathrm{mM} / \mathrm{l})\end{array}$} & $\geq 0.9{ }^{\circledR}$ & 5 & 20 & 8.00 & $2.51-25.51$ & $P=0.0004^{*}$ \\
\hline & $<0.9$ & 30 & 15 & & & \\
\hline \multirow{2}{*}{$\begin{array}{l}\text { GSSG } \\
(\mathrm{mg} / \mathrm{ml})\end{array}$} & $<9.9 \AA$ & 5 & 28 & 24 & $6.82-84.43$ & $P=0.0011^{*}$ \\
\hline & $\geq 9.9$ & 30 & 7 & & & \\
\hline \multirow{2}{*}{$\begin{array}{l}\text { GSH } \\
(\mathrm{mg} / \mathrm{ml})\end{array}$} & $\geq 17.30 ®$ & 0 & 27 & 22.97 & $12.70-41.55$ & $P=0.0002^{*}$ \\
\hline & $<17.30$ & 35 & 8 & & & \\
\hline \multirow{2}{*}{$\begin{array}{l}\text { Glutathione redox status } \\
\text { (GSH/GSSG) }\end{array}$} & $\geq 1.70 \AA$ & 2 & 32 & 17.60 & $7.56-23.11$ & $P=0.0001^{*}$ \\
\hline & $<1.70$ & 33 & 3 & & & \\
\hline
\end{tabular}

$\mathrm{n}$ - Sample size; ${ }^{\circledR}$ - reference group; $\mathrm{Cl}$ - Confidence interval; ${ }^{*}$ - Significance was considered at $P$-value $<0.05$

one showed a significant AUC (70.5), $(p=0.035)$, with sensitivity (84.6\%) and specificity $(80.6 \%)$ at a cut-off value $(9.9 \mathrm{mg} / \mathrm{dl})$. Serum levels of reduced glutathione showed a significant AUC (92.9), $(p=0.000)$, with sensitivity (92.9\%) and specificity (100\%) at a cut-off value $(17.3 \mathrm{mg} / \mathrm{dl})$. Serum levels of glutathione redox status (GSH/GSSG) showed a significant AUC (82.1), $(p=0.001)$, with sensitivity (92.9\%) and specificity $(76.9 \%)$ at a cut-off value (1.70).

\section{The association of serum levels of oxidative stress biomarkers with the risk of breast cancer incidence}

The values of odds ratio and confidence interval at certain cut-off values for serum levels of oxidative stress biomarkers were shown in Table 3.

\section{Correlation of serum levels of oxidative stress biomarkers with breast cancer clinicopathological data}

The results of the current study showed that none of the assayed biomarkers had a significant correlation with anyone of breast cancer clinicopathological features.

\section{Discussion}

Oxygen-free radicals (OFR), generated by a number of processes in vivo, are highly reactive and toxic [14]. However, biological systems have evolved an array of enzymatic and non-enzymatic antioxidant defence mechanisms to combat the deleterious effects of OFR. Reduced glutathione (GSH), in conjunction with glutathione peroxidase (GPx) and glutathione S-transferase (GST), plays a central role in the defence against free radicals, peroxides and a wide range of xenobiotics and carcinogens $[15,16]$.

Oxidative stress arises when there is an imbalance between OFR formation and scavenging by antioxidants. Excessive production of OFR can cause oxidative damage to biomolecules resulting in lipid peroxidation, mutagenesis and carcinogenesis. OFR-induced lipid peroxidation has been implicated in neoplastic transformation. Damage to the breast epithelium by OFR can lead to fibroblast proliferation, epithelial hyperplasia, cellular atypia and breast cancer [17].

In the current study, the serum levels of MDA were found to be significantly elevated in breast cancer patients than controls. As MDA is considered a marker for lipid peroxidation, this means that lipid peroxidation may play a role in breast carcinogenesis. Our results were in agreement with Rajneesh et al.,2008 [17].

Regarding the effect of MRM on the serum levels of MDA, the current study revealed significantly decreased levels of MDA after two weeks of surgery compared with its levels before surgery; this means that serum MDA can be used as an indicator for the effect of MRM on breast cancer patients. With respect to the effect of chemotherapy on the serum levels of MDA, our results showed a non-significant increase in serum MDA levels after 6 cycles of adjuvant combination chemotherapy compared with its levels after 2 weeks of surgery.

Total antioxidant capacity (TAC) measures the peroxyl-scavenging capacity of the extracellular antioxidant system that is comprised of sulphydryl groups, urate, ascorbate, carotenoids, retinol, $\alpha$-tocopherol, bilirubin and proteins. TAC reflects residual antioxidant capacity 
after the consumption of reactive oxygen species [18, 19]. In the current study, the serum levels of TAC were found to be significantly reduced in breast cancer patients before surgery than controls, this means that TAC may have a protective role against breast carcinogenesis. Our results supported those of Mahmood et al., 2009 [20] On the other hand, neither MRM nor chemotherapy has a significant effect on the serum levels of TAC.

Regarding the serum levels of GSH, the results of the current study showed significantly declined levels of GSH in breast cancer patients compared with normal healthy controls. This means that serum GSH can be used to differentiate breast cancer patients from controls. The results of the current study were in agreement with the findings of Yeh et al. [21] who reported that the levels of GSH were significantly decreased in the blood of breast cancer patients compared with controls. Yeh et al. [21] suggested that this could be due to both the increased GSH detoxification capacities, which can lead to GSH depletion within the red blood cells, and lower efficacy in the reduction of GSSG to GSH. These findings support the idea of the protective role of GSH against reactive oxygen species-mediated oxidative stress in cancer patients. At the same time, Yeh et al. [21] proposed that GSH should be regarded as an important biomarker for detecting breast malignancy.

With respect to the effect of surgery on serum levels of $\mathrm{GSH}$, the results of the current study showed that the serum levels of GSH were significantly increased in breast cancer patients after two weeks of MRM compared with their levels before surgery. This means that the serum levels of GSH can be used to monitor the effect of MRM on breast cancer patients. The findings of the current study can be explained according to Navarro et al. [22] who suggested that the tumour mass acts as a source for peroxides that reacts with and consume GSH leading to depletion of GSH. On this base, removal of the tumour decreases these peroxides resulting in an increase in GSH content which is in agreement with the results of the current study. With reference to the effect of six cycles of chemotherapy, the results of the current study showed a non-significant effect of chemotherapy on the serum levels of GSH compared with their levels after two weeks of MRM. This means that GSH cannot be used to monitor the response of breast cancer patients to chemotherapy.

Regarding the serum levels of GSSG and the glutathione redox status (GSH/GSSG), the results of the current study indicated significantly elevated levels of GSSG and significantly decreased levels of glutathione redox status (GSH/GSSG) in the serum of breast cancer patients than controls. These findings were in accordance with the results of Navarro et al. [22] who reported significantly higher levels of GSSG and decreased levels of glutathione redox status (GSH/GSSG) in breast cancer patients compared with controls. In their study, Navarro et al. [22] postulated that during cancer growth, the glutathione redox status (GSH/GSSG) decreases in the blood due to an increase in GSSG levels. Two reasons may explain the increase in blood GSSG: (a) the increase in peroxide production by the tumour that can lead to GSH oxidation within the red blood cells; and (b) an increase of GSSG release from different tissues into the blood.

With respect to the effect of surgery on the serum levels of GSSG and glutathione redox status (GSH/GSSG), the results of the current study showed that the serum levels of GSSG were significantly decreased, with no significant effect on glutathione redox status (GSH/GSSG), in breast cancer patients after two weeks of MRM compared with their levels before surgery. This means that the serum levels of GSSG can be used to monitor the effect of MRM on breast cancer patients. The findings of the current study can be also explained according to Navarro et al. [22] who suggested that the tumour mass acts as a source for peroxides that reacts with GSH resulting in the formation of GSSG. When the tumour mass is removed the amount of these peroxides decreases resulting in a decrease in the amount of GSSG formed due to the detoxification reaction. With reference to the effect of six cycles of chemotherapy, the results of the current study showed non-significant effects of chemotherapy on the serum levels of GSSG and glutathione redox status (GSH/GSSG) compared with their levels after two weeks of MRM. This means that none of these biomarkers can be used to monitor the response of breast cancer patients to chemotherapy.

The significant elevation in the serum levels of MDA and GSSG and the significant decline in the serum level of TAC, GSH and glutathione redox status (GSH/GSSG) in breast cancer patients before surgery compared to normal controls suggested the possibility of using anyone of these biomarkers for prediction of breast cancer to differentiate the breast cancer patient from the normal healthy controls. This directed us to compare the diagnostic accuracy of these biomarkers to determine which of them has the highest and the lowest diagnostic value. This comparison was carried out using the ROC curve analysis in such a way that the greater area under the ROC curve corresponds to a better diagnostic test. Serum levels of MDA showed the greatest significant area under the curve (96\%) followed by GSH (92.9\%), glutathione redox status (GSH/GSSG) $(82.1 \%)$, TAC (76.9\%) then GSSG $(70.5 \%)$. These results suggested that serum levels of MDA are superior to GSH, GSH/GSSG, TAC and GSSG for diagnosis of breast cancer patients. The results of the current study supported those reported by Pande et al. [23] who found that the diagnostic accuracy of serum MDA 
was superior to TAC for distinguishing breast cancer patients from controls

Regarding the association of the assayed biomarkers with the risk of breast carcinogenesis, the results of the current study illustrated that increased levels of serum MDA and GSSG and decreased levels of TAC, GSH and glutathione redox status can significantly potentiate breast cells for malignancy. Pande at al., 2012 [23].

From the current study, it could be concluded that all of the assayed biomarkers have a diagnostic role in breast cancer patients with MDA and GSH being superior to the other biomarkers for discrimination of breast cancer patients from controls. Serum levels of MDA, GSH and GSSG were good indicators for monitoring the effect of surgery. All the assayed biomarkers were found to be associated with breast cancer risk. None of the assayed biomarkers could predict the effect of chemotherapy.

\section{References}

1. Schieber M, Chandel NS. ROS function in redox signaling and oxidative stress. Curr Biol. 2014; 24(10): R453-R462, doi: 10.1016/j. cub.2014.03.034, indexed in Pubmed: 24845678.

2. Tas $\mathrm{F}$, Hansel $\mathrm{H}$, Belce $\mathrm{A}$, et al. Oxidative stress in breast cancer. Med Oncol. 2005; 22(1): 11-15, doi: 10.1385/MO:22:1:011, indexed in Pubmed: 15750191

3. Sharhar S, Normah $\mathrm{H}$, Fatimah A, et al. Antioxidant intake and status and oxidative stress in relation to breast cancer risk: a case-contro study. Asian Pac J Cancer Prev. 2008; 9(2): 343-349, indexed in Pubmed: 18712988.

4. Kang DH. Oxidative stress, DNA damage, and breast cancer. AACN Clin Issues. 2002: 13(4): 540-549, doi: 10.1097/00044067-20021100000007, indexed in Pubmed: 12473916.

5. Rajneesh CP, Manimaran A, Sasikala KR, et al. Lipid peroxidation and antioxidant status in patients with breast cancer. Singapore Med J. 2008; 49(8): 640-643, indexed in Pubmed: 18756349.

6. Panis C, Victorino VJ, Herrera AC, et al. Can Breast Tumors Affect the Oxidative Status of the Surrounding Environment? A Comparative Analysis among Cancerous Breast, Mammary Adjacent Tissue, and Plasma. Oxid Med Cell Longev. 2015; 2015: 6429812, doi: 10.1155/2016/6429812, indexed in Pubmed: 26697139
7. Zitka O, Skalickova S, Gumulec J, et al. Redox status expressed as GSH:GSSG ratio as a marker for oxidative stress in paediatric tumour patients. Oncol Lett. 2012; 4(6): 1247-1253, doi: 10.3892/ol.2012.931, indexed in Pubmed: 23205122.

8. Haskell CM, Lowitz BB, Casciato AD. Breast cancer. In: Casciato $A D$ and ; eds. Manual of clinical oncology; 2nd ed. Little and Brown Company, Boston, Toronto (pub. ) 1985, pp. : 150-65.

9. Rintoul RF. Operations on the breast. In: Farquhaerisons text book of operative surgery; 7th ed. Churchill living stone 1986: 270-81.

10. Abeloff MD, Lichter AS, Niederhuber JE, Pierce LJ, Aziz DC. Breast. Abeloff MD, Armitage JO, Licher AS, Niederhuber JE. ed. Clinical Oncology. Churchil livingstone Inc. 1995: 1617-1714.

11. Griffith OW. Determination of glutathione and glutathione disulfide using glutathione reductase and 2-vinylpyridine. Anal Biochem. 1980; 106(1): 207212, doi: 10.1016/0003-2697(80)90139-6, indexed in Pubmed: 7416462.

12. Zitka O, Skalickova S, Gumulec J, et al. Redox status expressed as GSH:GSSG ratio as a marker for oxidative stress in paediatric tumour patients. Oncol Lett. 2012; 4(6): 1247-1253, doi: 10.3892/ol.2012.931, indexed in Pubmed: 23205122

13. Kirlin WG, Cai J, Thompson SA, et al. Glutathione redox potential in response to differentiation and enzyme inducers. Free Radic Biol Med. 1999; 27(11-12): 1208-1218, doi: 10.1016/s0891-5849(99)00145-8, indexed in Pubmed: 10641713

14. Marnett L. Oxyradicals and DNA damage. Carcinogenesis. 2000; 21(3): 361-370, doi: 10.1093/carcin/21.3.361.

15. Datta K, Sinha S, Chattopadhyay P. Reactive oxygen species in health and disease. Natl Med J India. 2000; 13(6): 304-310, indexed in Pubmed: 11209486

16. Matés JM, Pérez-Gómez C, Núñez de Castro I. Antioxidant enzymes and human diseases. Clin Biochem. 1999; 32(8): 595-603, doi: 10.1016/s0009-9120(99)00075-2, indexed in Pubmed: 10638941.

17. Rajneesh CP, Manimaran A, Sasikala KR, et al. Lipid peroxidation and antioxidant status in patients with breast cancer. Singapore Med J. 2008; 49(8): 640-643, indexed in Pubmed: 18756349.

18. Wayner DD, Burton GW, Ingold KU, et al. The relative contributions of vitamin $\mathrm{E}$, urate, ascorbate and proteins to the total peroxyl radical-trapping antioxidant activity of human blood plasma. Biochim Biophys Acta. 1987; 924(3): 408-419, doi: 10.1016/0304-4165(87)90155-3, indexed in Pubmed: 3593759.

19. Wang XL, Rainwater DL, VandeBerg JF, et al. Genetic contributions to plasma total antioxidant activity. Arterioscler Thromb Vasc Biol. 2001: 21(7): 1190-1195, doi: 10.1161/hq0701.092146, indexed in Pubmed: 11451750

20. Mahmood IH, Abdullah KS, Abdullah MS. Total antioxidant status in women with breast cancer. Pak J Med Sci. 2009; 25: 609-12.

21. Yeh CC, Hou MF, Wu SH, et al. A study of glutathione status in the blood and tissues of patients with breast cancer. Cell Biochem Funct. 2006; 24(6): 555-559, doi: 10.1002/cbf.1275, indexed in Pubmed: 16142688

22. Navarro J, Obrador E, Carretero J, et al. Changes in glutathione status and the antioxidant system in blood and in cancer cells associate with tumour growth in vivo. Free Radical Biology and Medicine. 1999; 26(34): 410-418, doi: 10.1016/s0891-5849(98)00213-5

23. Pande D, Negi R, Karki K, et al. Oxidative damage markers as possible discriminatory biomarkers in breast carcinoma. Transl Res. 2012; 160(6): 411-418, doi: 10.1016/j.trsl.2012.07.005, indexed in Pubmed: 22885175 